\title{
V404 Cyg: an INTEGRAL and Swift X-ray view of an obscured microquasar
}

\author{
S.E. Motta ${ }^{1}$, C. Sánchez-Fernández ${ }^{2}$, J. J. E. Kajava ${ }^{3,4}$ \\ ${ }^{1}$ University of Oxford, Department of Physics, Astrophysics, Denys Wilkinson Building, Keble \\ Road, Oxford OX1 3RH, UK \\ ${ }^{2}$ European Space Astronomy Centre (ESA/ESAC), Science Operations Department, 28691 \\ Villanueva de la Cañada, Madrid, Spain \\ ${ }^{3}$ Finnish Centre for Astronomy with ESO (FINCA), University of Turku, Väisäläntie 20, \\ FIN-21500 Piikkiö, Finland \\ ${ }^{4}$ Tuorla Observatory, University of Turku, Väisäläntie 20, FIN-21500 Piikkiö, Finland \\ E-mail: sara.motta@physics.ox.ac.uk
}

\begin{abstract}
The black hole X-ray binary V404 Cyg entered an outburst phase on 2015 June 15 after 26 years of X-ray quiescence. Its activity was monitored at all wavelengths by virtually all the ground and space based facilities that could observe the source. This produced the largest multi-wavelength dataset on an active black hole X-ray transient. Here we focus on the X-ray monitoring campaign we carried out with the INTEGRAL and Swift satellites. We briefly summarize the results that we obtained from the observation of the most active phase of the outburst and we will describe our understanding of the source in the context of what it is known about accreting stellar mass and super-massive black holes. The results of time-resolved spectroscopy revealed that while the soft X-ray spectra extracted from Swift data reveal the effects of very variable, high columndensity material obscuring the source, the hard X-ray spectra extracted from INTEGRAL are most sensitive the the source intrinsic (i.e. accretion driven) changes. Our results suggest that the inner part of the accretion flow in V404 Cyg is inflated into a so-called slim disk that hides the innermost regions of the flow, but also produces a cold, inhomogeneous, high-density outflow that introduces the high-absorption and fast spectral variability. We argue that the black hole in V404 Cyg might have been accreting erratically or even continuously at Eddington/Super-Eddington rates, while being partly or completely obscured. We therefore conclude that the part or even all of the flaring behaviour of the source might have not been accretion-driven, but rather the result of the covering/unveiling of the extremely bright source hidden within the system.
\end{abstract}

11th INTEGRAL Conference Gamma-Ray Astrophysics in Multi-Wavelength Perspective,

10-14 October 2016

Amsterdam, The Netherlands

* Speaker. 


\section{Introduction}

Most black hole (BH) X-ray binaries (BHBs) are transient systems that alternate between relatively short outbursts and long periods of (X-ray) quiescence. Transient BHBs typically show significant luminosity variations, from $L \sim 10^{30-31} \mathrm{erg} \mathrm{s}^{-1}$ in quiescence to $L \sim 10^{38-39} \mathrm{erg} \mathrm{s}^{-1}$ or more in outburst. During outburst, cyclic "hysteresis" patterns are easily observed in the socalled Hardness-Intensity diagram (HID; see e.g., [1]), which have been shown to have a clear and repeatable association with mechanical feedback in the form of winds and relativistic jets $[2,3]$.

Transient BHBs show different spectral states, associated to different areas of the HID. In the hard state (LHS; right side of the HID, see e.g. [4]) the X-ray spectrum is dominated by emission from Compton up-scattering of soft seed photons which are either produced in a cool geometrically thin accretion disk likely truncated at large radii, by synchrotron-self-Compton emission from hot electrons located close to the central $\mathrm{BH}$ [5], or by synchrotron emission from a compact jet [6]. In the soft state (HSS; left areas of the HID) the X-ray spectrum is dominated by thermal emission from a geometrically thin accretion disk extending down to the innermost stable circular orbit around the BH. In between these two states lie the so-called intermediate states (hard and soft intermediate states, HIMS and SIMS, respectively), where the X-ray spectra show properties characteristic of both the LHS and HSS as it evolves during the spectral transitions between the LHS and HSS. On rare occasions, some systems may also show a so-called ultra-luminous state [7] (also known as the very-high state), characterized by very high luminosities and an "intermediate" spectrum. An iron K- $\alpha$ emission line with variable equivalent width at $\sim 6.4 \mathrm{keV}$ can be detected in all states, together with a reflection hump peaking at energies around $30 \mathrm{keV}$.

While most BHBs accreting below the Eddington limit fit into this picture, a few systems accreting at extreme rates do not. Typical examples are the BHBs GRS 1915+105, which has been accreting close to Eddington for most of its (still ongoing) 23-years long outburst, and V4641 Sgr which in 1999 showed a giant outburst associated to a super-Eddington accretion phase [8]. While GRS 1915+105 displays relatively soft spectra, V4641 Sgr showed significant reflection and heavy and variable absorption that have been ascribed to an extended optically thick envelope/outflow ejected by the source $[8,9]$.

When the accretion rate approaches or exceeds the Eddington limit, the radiative cooling time scale to radiate all the dissipated energy locally (a key requirement for thin disks) becomes longer than the accretion time scale. Therefore, the radiation is trapped and advected inward with the accretion flow, and consequently both the radiative efficiency and the observed luminosity decrease. This configuration is known as slim disk $[10,11]$. The slim disk model has been successfully applied to stellar mass BHs, such as the obscured BH candidate SS 433 [12], to ultraluminous X-ray sources [13], and to super massive BHs (narrow-line Seyfert galaxies, e.g. [14]).

\subsection{V404 Cygni}

The first X-ray outburst from V404 Cyg was observed by Ginga in 1989 [15]. The system orbital period is $\sim 6.5$ days and the system mass function $\left(f(\mathrm{M})=6.26 \pm 0.31 \mathrm{M}_{\odot}\right)$, confirmed the $\mathrm{BH}$ nature of the compact object in V404 Cyg, classifying it as a low-mass X-ray binary [16]. The distance $d=2.39 \pm 0.14 \mathrm{kpc}$ to the source is measured through radio parallax [17]. 
On 2015 June 15 (MJD 57188), Swift/BAT triggered on a bright hard X-ray source, which was soon recognized to be V404 Cyg in outburst after 26 years of quiescence [18, 19]. After the initial Swift/BAT trigger, INTEGRAL observed V404 Cyg almost continuously during the entire outburst, providing the best hard-X-ray coverage ever obtained for this source [20, 21]. Swift provided instead several short observations all along the outburst. The source reached the outburst peak on June 26 and then began a rapid fading towards X-ray quiescence (reached between 2015 August 5 and August 21, [22]). Throughout this outburst V404 Cyg displayed highly variable multi-wavelength activity (e.g., $[23,24,25,26])$, that was monitored by the astronomical community through one of the most intensive observing campaigns performed on an X-ray binary outburst to date (see [22], and references therein).

Here we briefly review the results obtained from the time-resolved spectral analysis that we performed on Swift/XRT and INTEGRAL/IBIS/ISGRI data. We analyzed the the observations taken during the outburst most active phase, roughly corresponding to the first two weeks of activity after the outburst onset (see Fig. 1). We concentrate on the variations of the source spectral properties induced by the presence of a variable neutral absorber local to the source, and on the variability of the source hard X-ray spectrum, which shows slower and and likely accretion driven changes in the source. Results from the analysis of INTEGRAL/IBIS/ISGRI data were reported by [27]. Results based on the Swift/XRT observations were presented by Motta et al. 2017c. Results of the spectral analysis of a small INTEGRAL/JEM-X, INTEGRAL/IBIS/ISGRI and Swift/XRT simultaneous dataset have been reported by [28].

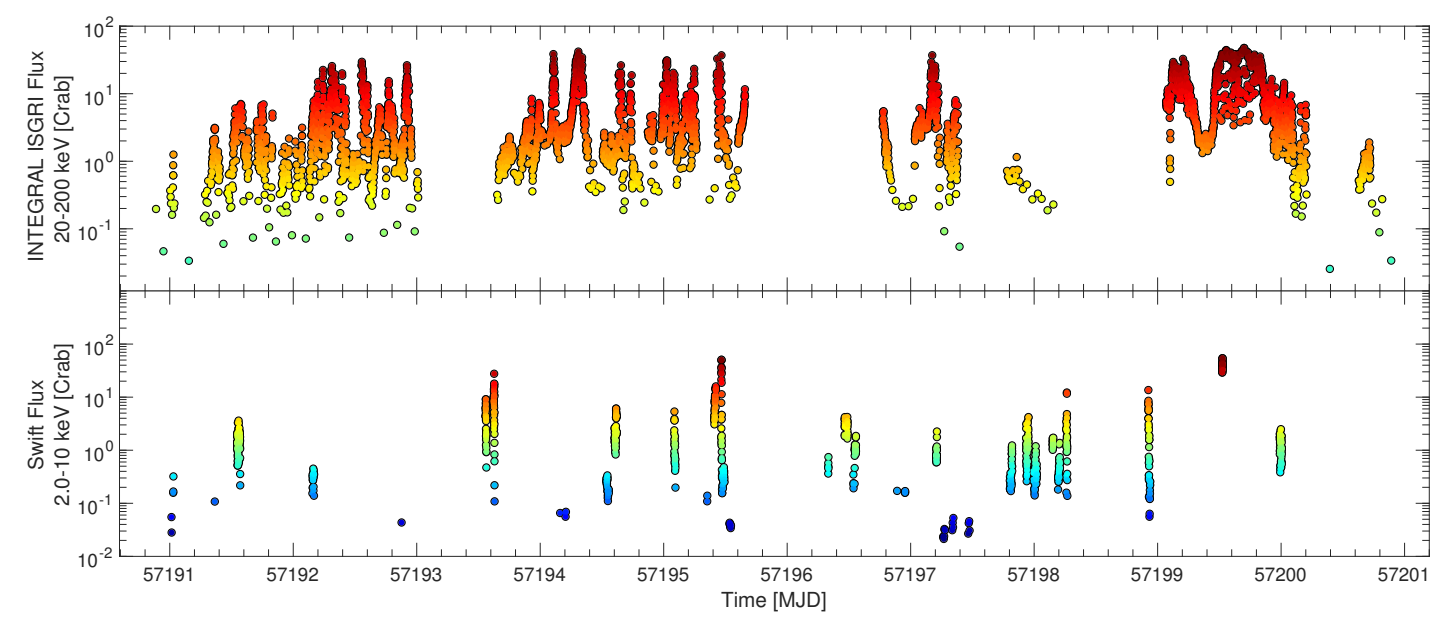

Figure 1: Top panel: INTEGRAL/IBIS/ISGRI light-curve of of V404 Cyg during the flaring phase of the June-August 2015 outburst (20-200 keV energy range). The count rate has been converted to Crab units using the Crab count rates obtained during INTEGRAL revolutions 1598 and 1599. Each point corresponds to a $64 \mathrm{~s}$ long bin. Bottom panel: Swift/XRT light-curve of the flaring phase of the 2015 June-August outburst. In both panels, the points are colour-coded according to the flux, with redder points corresponding to higher fluxes. Each point corresponds to a variable exposure stretch of data, whose time duration has been determined based on the source flux (see [27] and Motta et al. 2017c for details). Note that the flux scale is different in the two panels due to the different energy bands of INTEGRAL/ISGRI and Swift/XRT. 


\section{A look to the variability of the high-energy spectral continuum}

The intense flaring seen in INTEGRAL/IBIS/ISGRI light curves of V404 Cyg during the June 2015 outburst are not typical features of standard BHB outbursts(e. g. [29]). Similarly, the soft $\mathrm{X}$-ray spectra are remarkably different from the spectra of other BHBs, mostly owing to extreme intrinsic absorption affecting V404 Cyg (see Motta et al. 2017c and Sec. 3), already observed during the 1989 outburst in the data collected by Ginga ([30]). However, the hard X-rays spectra (above $20 \mathrm{keV}$ ) clearly show similarities with what is seen in well-behaved BHBs.

We performed time-resolved analysis of IBIS/ISGRI spectra with variable exposure times (from tens to thousands of seconds). We fitted the spectra in the 20-200 keV energy range using spectral models based on thermal Comptonization (NTHCOMP and COMPPS; see [27] for details), depending on the following spectral parameters: the spectral slope, $\Gamma$, electron temperature, $T_{e}$, optical depth, $\tau$, and column density, $N_{\mathrm{H}}$. We also measured the X-ray flux $F_{\mathrm{X}}$ in the 20-200 keV energy band from every spectrum we fitted. Using these parameters, we built a $F_{\mathrm{X}}-\Gamma$ diagram, that essentially corresponds to the HID typically used to describe BHBs in outburst. We identified two clear spectral branches in our diagram, a hard branch and a soft flaring branch, that share similarities with the canonical BHB hard state and intermediate state, respectively.

In the hard branch, V404 Cyg shows a hard thermal Comptonized spectrum, which softens as the flux increases. While $\tau$ is correlated with $F_{\mathrm{X}}, T_{\mathrm{e}}$ is anti-correlated with $F_{\mathrm{X}}$ and $\Gamma$. These correlations are characteristic of the BHB hard state [27]. This parameter evolution can be explained in terms of thermal Comptonization of soft seed photons by a hot electron distribution close to the $\mathrm{BH}$. The $F_{\mathrm{X}}-T_{\mathrm{e}}$ anti-correlation could arise due to the electron population cooling as more disk photons enter the Comptonizing medium when the inner accretion disk edge moves closer to the BH.

In the soft flaring branch V404 Cyg is characterized by soft, thermal Comptonized spectra, which show softening with increasing flux. Contrarily to the hard branch, in the soft branch $T_{\mathrm{e}}$ and $F_{\mathrm{X}}$ are correlated, while $F_{\mathrm{X}}$ and $\tau$ are anti-correlated. This behaviour is very similar to what is observed during the intermediate state and occasionally the ultra-luminous state in other BHBs.

The IBIS/ISGRI data also shows a number of X-ray plateaus characterised by roughly constant fluxes. The Comptonization models fail to describe the source spectra during these plateaus if the column density was fixed to the interstellar values in the direction of the source. These spectra occupy characteristic regions in the $F_{\mathrm{X}}-\Gamma$ diagram, which we call the plateau branch. We found that fitting these spectra requires column densities around $N_{\mathrm{H}} \gtrsim 5 \times 10^{24} \mathrm{~cm}^{-2}$, suggesting that the observed drops in hard X-ray emission were mainly due to obscuration of the central parts of the source by Compton-thick material local to the source.

\section{Probing the effects of a clumpy absorber outflowing from the source}

Spectral variability on times scales down to a few tens of seconds can be seen from the Swift/XRT spectra collected during the most active phase of the June 2015 outburst of V404 Cyg. Variations in flux as large as 3 orders of magnitude in time-spans as short as a few minutes are also detected. This extreme variability could be explained by assuming that a thick layer of non- 

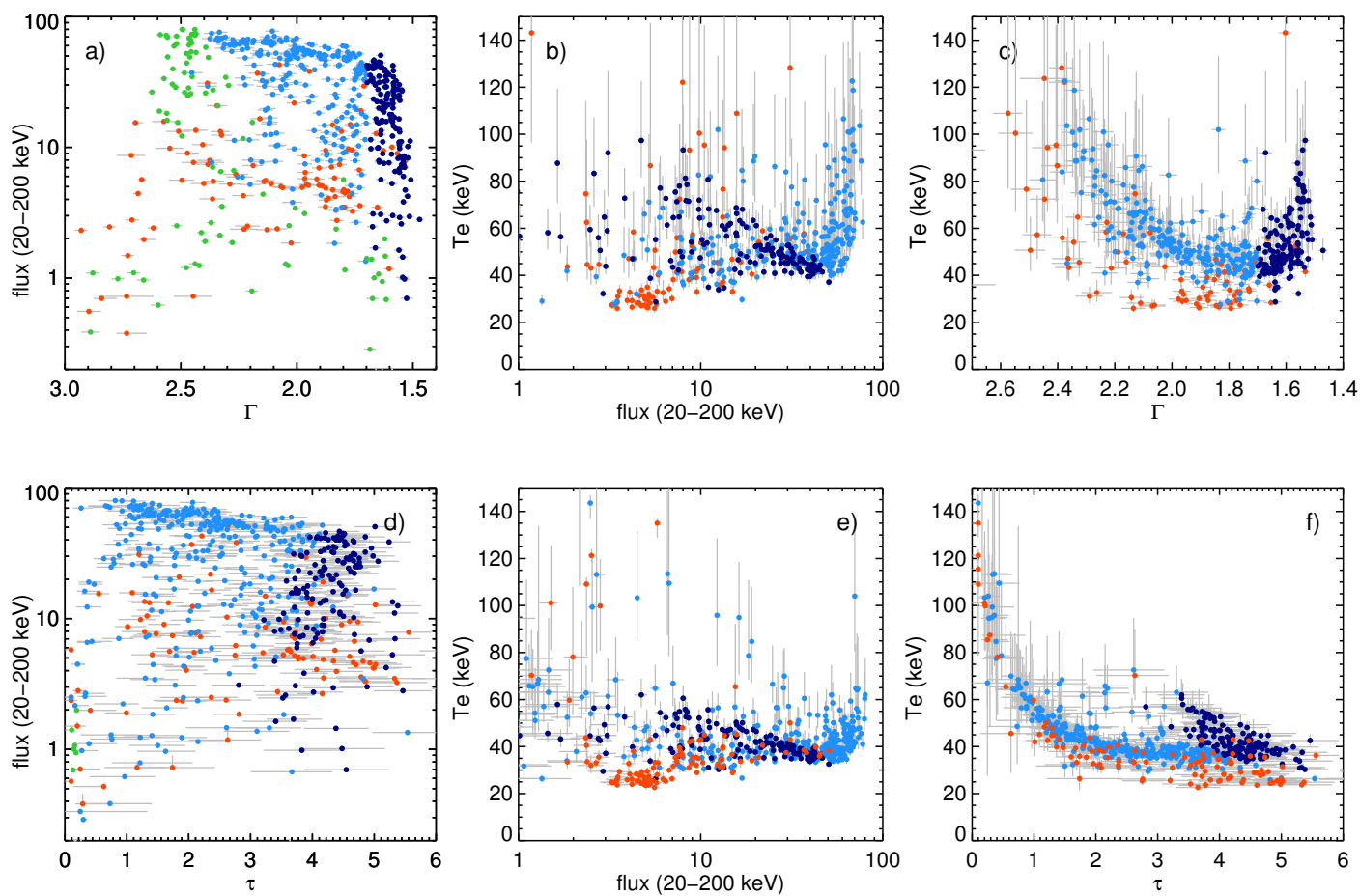

Figure 2: Relations between the spectral parameters derived from the fits to the IBIS/ISGRI spectra of V404 Cyg using NTHCOMP (panels a-c) and COMPPS (pannels d-f). Green, blue and red symbols highlight different best-fitting models. Blue: Comptonization models with constrained electron temperature $T_{\mathrm{e}}$, further divided into hard ( $\Gamma \leq 1.7$; dark blue) and soft spectra $(\Gamma>1.7$; light blue). Green: Comptonization models with unconstrained $T_{\mathrm{e}}$. Red: spectra that could not be well-fit by a Comptonization models. Panel a) $F_{\mathrm{X}}-\Gamma$ diagram. We find a hard branch (dark blue points) where $F_{\mathrm{X}}$ and $\Gamma$ are correlated, similar to the hard state in the BHB HID. At the highest fluxes in the outburst, we find the soft flaring branch (light blue points), similar to the BHB HID intermediate states. We also identify the softest spectra in our sample $(\Gamma \gtrsim 2.4)$ with unconstrained $T_{\mathrm{e}}$ with a tentative ultra-luminous state (green points). The different branches also occupy characteristic regions in panel d) $F_{\mathbf{X}}-\tau$ diagram, panels b, e), $F_{\mathbf{X}}-T_{\mathrm{e}}$ diagrams, panel $\left.\mathbf{c}\right) \Gamma-T_{\mathrm{e}}$ diagram and panel f) $\tau-T_{\mathrm{e}}$ diagram. (From [27]).

uniform, high-column density material local to the source is causing the dramatic spectral and flux changes.

\subsection{The outflowing absorber}

Our analysis showed that the spectral changes occurring on short time-scales ( $<$ minutes) can be ascribed to the variations of the material covering locally the source. While variations in the column density may cause fast ( $\sim$ minutes) but moderate flux changes, variations in the covering fraction are instead responsible for the most dramatic flux and spectral changes. This behaviour was already observed in the Ginga data collected during the 1989 outburst (see e.g. [31]).

We found that the absorber local to the source has a bi-modal behaviour: it appears to be either very dense (possibly even Compton-thick, with the column larger than the inverse of the Thomson cross-section $N_{\mathrm{H}} \geq \sigma_{T}^{-1} \simeq 1.5 \times 10^{24} \mathrm{~cm}^{-2}$ ) and highly inhomogeneous/clumpy, or tenuous and 
uniform (see Fig. 3, panels a, b and k). This suggests that two types of absorber could be present at all times, i.e. one inhomogeneous and high-density, and one uniform and tenuous. The $S$ wift data were not sensitive to the effects of the former unless the latter was not present. Furthermore, it is possible that the uniform, thin absorber is located at a large distance from the central $\mathrm{BH}$ with respect to the clumpy absorber, and could even correspond to the neutral outflowing material launched as an optical wind at hundreds of thousands of $R \mathrm{~g}$ from the central BH [32]. We also found that there is a clear link between the strongest flares seen by both INTEGRAL and Swift, and drops in column density. In particular, sudden drops in the local column density and simultaneous disappearance of partial covering appear to be related to dramatic increases in flux. In this context, the super-Eddington flares observed in V404 Cyg would correspond to times where the actual source emission (often or even always close to Eddington) reaches the observer unabsorbed, while the lower flux phases would be explained by partial or complete covering of the most intense central X-ray source.

Our spectral analysis revealed the presence of an emission line only at phases of high column density, indicating that the line might be produced by the same material that is causing the absorption. Interestingly, the iron-K $\alpha$ line shows significant blue and sometimes red shifts (up to $7.2 \mathrm{keV}$ and down to $6.2 \mathrm{keV}$, respectively), clearly suggesting that the material responsible for both the line and the variable absorption is outflowing from the source. Given that the inclination of the source is known with high precision $\left(i \approx 67^{\circ}\right)$, through the shift of the line we obtained an upper limit to the outflow velocity of $0.1 \mathrm{c}$, and to the outflow launching angle of $35^{\circ}$ from the disk plane.

\subsection{The intrinsic source spectrum}

In order to be able to launch a non-homogeneous outflow the accretion flow must sustained by strong radiation forces (consequence of high, possibly super-Eddington, accretion rates) forming a so-called slim disk [11], as opposed to a standard Shakura-Sunyaev thin disk [33]. Such slim disks are significantly vertically extended, and the launch angle that we inferred above therefore sets an upper limit to the slim disk half-opening angle. This implies that our line of sight passes straight through the slim disk/outflow, explaining the dramatic effects of the absorber on the source emission, and also the fact that we did not find any signature of the presence of a thin accretion disk in the Swift data.

The $S$ wift-based time resolved spectroscopy showed that V404 Cyg does not reflect the typical softer-when-brighter behaviour of other BHBs. Rather, the largest flares seen in V404 Cyg appear to correspond to spectral hardening. Motta et al. 2017c showed that the extremely hard spectra seen in the Swift data are likely significantly affected by reflection. Therefore, the unusual relation between photon index and flux observed in V404 Cyg in the soft X-ray band could be ascribed to the fact that the inner accretion flow cannot be described, as stressed above, by the standard ShakuraSunyaev thin disk typically observed in BHBs [33]. The innermost regions of the accretion flow, instead, most likely take the form of a slim disk that is able to launch a clumpy outflow. Given that, as noted above, the increases in flux (and thus spectral hardening) generally correspond to a drop in column density, it is likely that the photon index resulting from our spectral fits does not reflect the intrinsic spectral slope of the X-ray source. Rather the photon index we measured is an apparent one, and it describes a complex spectral shape resulting from the peculiar geometric configuration

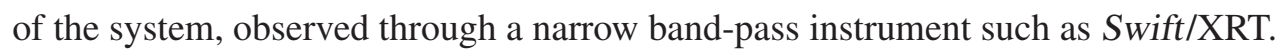






Figure 3: Parameter distributions, observed flux distribution, and parameters correlations from the best fits to the spectra of our sample using a partially-covered absorbed power law model in the form TBFEO $\times$ TBPCF $\times($ POWERLAW + GAUSS $)$. TBFEO and TBPCF account for the interstellar absorption (frozen to the value in the direction of the source, i.e. $N_{\mathrm{H} \text {,gal }}=8.3 \pm 0.8 \times 10^{21} \mathrm{~cm}^{-2}$ ) and for the absorption local to the source, respectively. POWERLAW is aimed at modelling the spectral continuum, while the GAUSS component describes the Iron-K $\alpha$ emission line observed in a number of spectra. This model depends on 6 parameters: the column density local to the source $N_{\mathrm{H}}$ and partial covering fraction PCF (from the TBPCF component), the power law slope $\Gamma$ and normalization $N_{\Gamma}$ (from the POWERLAW component), the line centroid energy $E_{\text {Line }}$ and normalization $N_{\text {Line }}$ (from the GAUSS component). From top to bottom (left column) and from left to right (bottom line) we show: column density local to the source (in log scale), partial covering fraction, photon index $\Gamma$, apparent flux (in log scale, corrected for the ISM absorption) and iron line energy. At the top of each distribution we report the mean value of each parameter. 


\section{Results from broad-band spectroscopy: signatures of heavy radiation reprocessing}

During our INTEGRAL and Swift monitoring campaign, we obtained a strictly simultaneous observation of V404 Cyg during one of the plateau phases described in Sec. 2, allowing us to analyse the broad-band X-ray energy spectrum of the system (Swift/XRT + INTEGRAL/JEM-X + INTEGRAL/IBIS/ISGRI, see Fig. 4). During this simultaneous observation, both the source flux and spectral shape were remarkably stable for $\approx 900$ seconds, contrarily to what was observed during most of the outburst, and we could obtain a high-quality average broad band X-ray spectrum of the source.

Modelling of the spectra based on the MYTORUS model (typically used to fit the spectra of partially obscured AGN, [34]) showed that the best description of the spectrum is given by a bright point source whose emission is scattered and reflected by a patchy toroidal reprocessor surrounding it. The illuminating spectrum could be well-described by a Compton spectrum with optical depth $\tau \sim 0.9$ and electron temperature $\approx 30 \mathrm{keV}$, consistent with the average electron temperature measured from INTEGRAL/ISBIS/ISGRI data (see Sec. 2 and [27]).

We found that the average column density of the toroidal reprocessor is in the Compton-thick regime $\left(N_{\mathrm{H}} \approx 3 \times 10^{24} \mathrm{~cm}^{-2}\right)$, while the column density in the direction of the line of sight is about a factor of four smaller $\left(N_{\mathrm{H}} \approx 0.8 \times 10^{24} \mathrm{~cm}^{-2}\right)$. This is expected in a scenario where a local, non uniform (patchy) and highly variable reprocessor heavily affects the emission from the central source. As the Comptonized emission, the transmitted emission and the scattered emission do not necessarily experience the same absorption. This can happen, for instance, in the situation where a Compton-thick clump of cold material is intercepting the line of sight. As expected in the presence of heavy absorption, the spectrum above $10 \mathrm{keV}$ is dominated by the reprocessed emission. A large fraction of such emission comes from photons scattered multiple times in the local absorber, while the remaining fraction is due to photons scattered through back-illuminated matter in the reprocessor, and to photons reflected off the far (with respect to the observer) inner side of the reprocessor and then reaching the observer without being further scattered.

We estimated that while the observed flux of the source was remarkably lower than its Eddington limit (corresponding to only a few percent of it), the intrinsic flux corresponded to a luminosity only slightly lower then the estimated Eddington luminosity for V404 Cyg. As mentioned earlier, the data considered corresponded to a plateau phase in the light curve of V404 Cyg [27] that occurred in between two of the major flares seen in the June 2015 outburst (flare peak observed on MJD 57194.11 and MJD 57194.31, respectively). The source flux measured during both such flares is consistent with the unabsorbed flux inferred from our modelling, indicating that the plateau phases were likely due to very high column density material temporarily obscuring almost completely the central X-ray engine [27].

\section{Discussion and conclusions: V404 Cyg as an obscured super-Eddington AGN-analogue}

We analysed the INTEGRAL and Swift data collected during the most active phase of the 2015 outburst of the BHB V404 Cyg (a.k.a. GS 2023+338) and performed time resolved spec- 


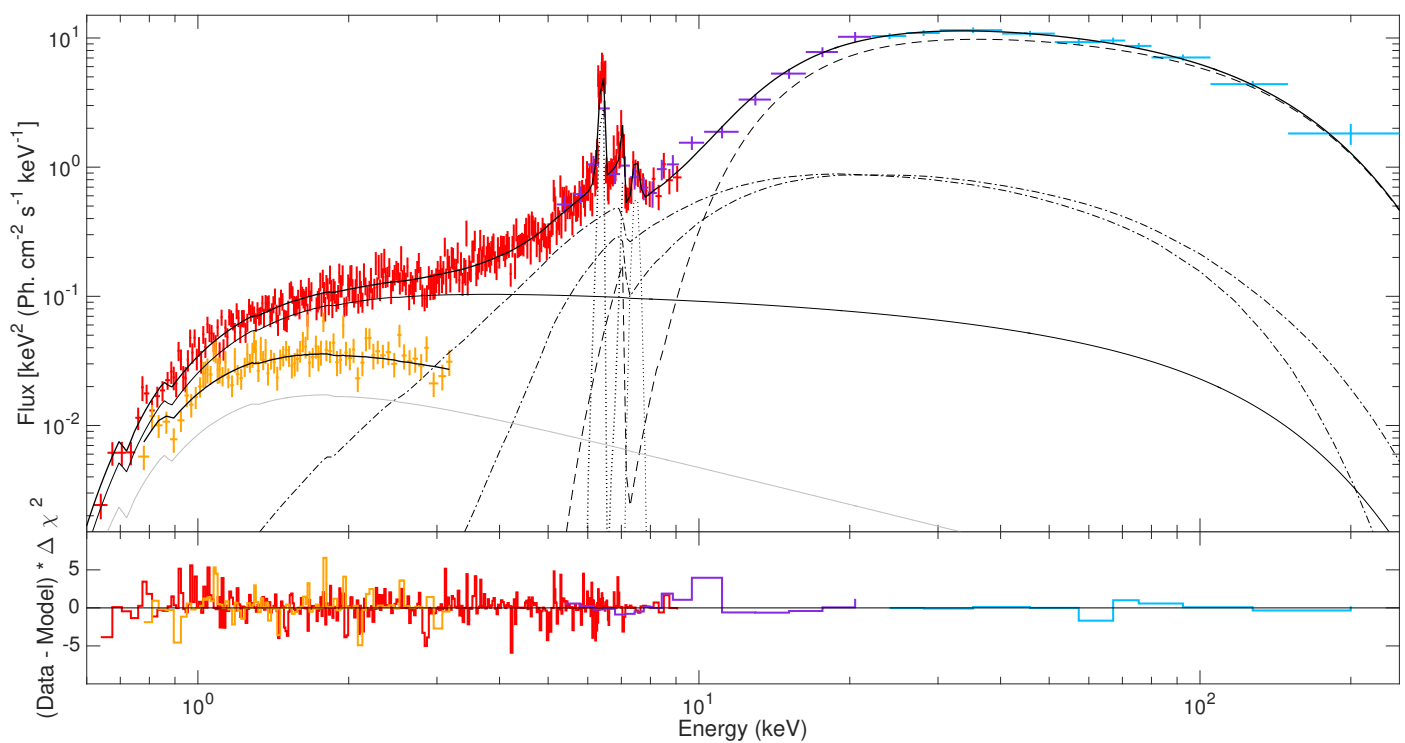

Figure 4: Figure taken from [28]. Top panel: Swift/XRT + INTEGRAL/JEM-X + INTEGRAL/ISGRI spectrum of V404 Cyg fitted a model based on MYTORUS (see [28] for details). Upper panel: Swift/XRT (red), INTEGRAL/JEM-X (purple), INTEGRAL/ISGRI (clear blue) source spectra and Swift/XRT dust scattering halo spectrum (orange). The thick black line marks the best fit to the data. The thin black line indicate the illuminating Compton continuum, the dashed black line marks the zeroth-order continuum (the emission that is transmitted to the observer after passing through the absorber), the dot-dashed lines mark the scattered continuum, the dotted line marks the fluorescent Fe-line spectra and the Ni-line at $\sim 7.5 \mathrm{keV}$. Finally, the solid grey line is the fit to the dust scattering halo spectrum (see $[28,35]$ ) as seen by Swift/XRT. Bottom panel: residuals to the model described above.

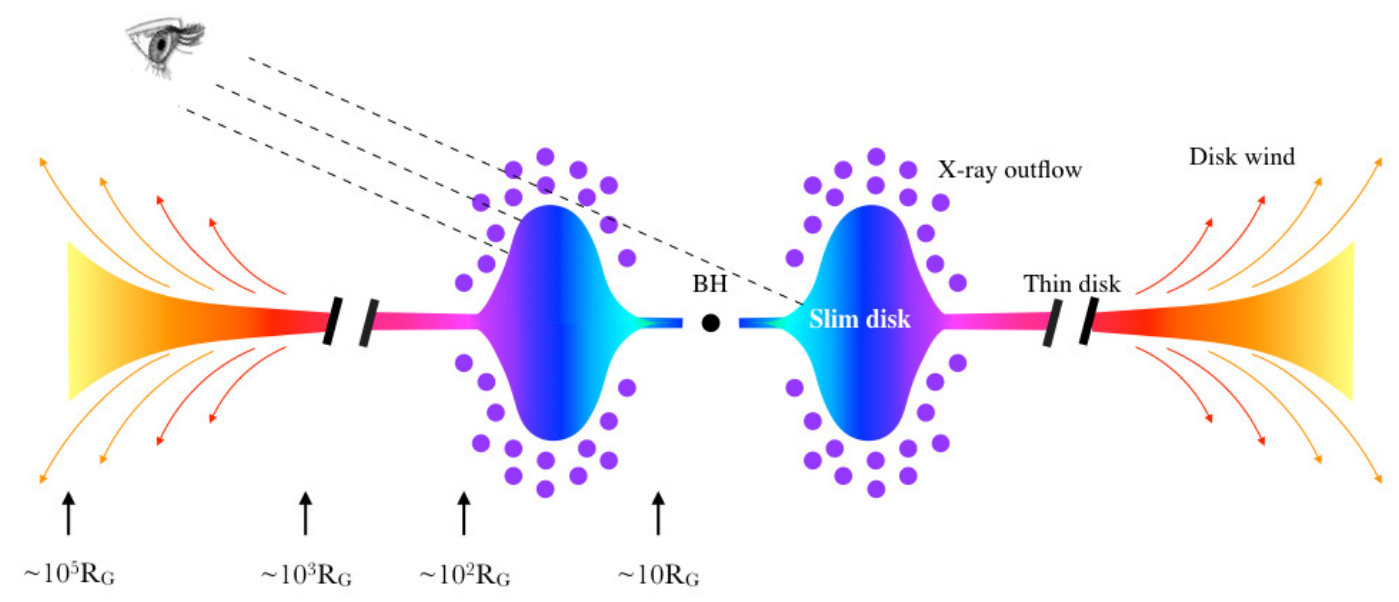

Figure 5: Figure taken from Motta et al. 2017c. Schematic view of V404 Cyg. The inner region of the accretion flow is inflated and originates the clumpy outflow that is responsible for the presence of the heavy, non-uniform layer of absorbing material local to the source. The outer disk, instead, is responsible for the launch of the strong thermal wind seen at optical wavelengths. A colour version of this Figure is available on-line. Note that slim disk in fact refers to an accretion-flow that has a significant vertical extent compared to a thin disk. 
troscopy on the $S$ wift low-energy data with very short time resolution (tens of seconds) and on the high-energy INTEGRAL data with longer exposures (tens to thousands seconds). We also performed broad-band time-averaged X-ray spectroscopy on a short simultaneous INTEGRAL and Swift observations taken during a phase when the source appeared to be weakly variable, but affected by heavy absorption and radiation reprocessing. The details of the analysis we carried out are reported in [28], [27] and Motta et al. 2017c.

We showed that the INTEGRAL data are most sensitive to the intrinsic variations of the source spectrum, particularly visible at high-energies where the effects of the high-column density local to the source are moderate. The Swift data, instead, mostly show the effects of a heavy and inhomogeneous absorber, whose changes are responsible for most - if not all - the fast spectral variability observed in the data. Such absorber is also responsible for the reflection features that we detected in the data. The INTEGRALand $S$ wift data together show that while the soft X-ray energy band display clear effects of heavy and inhomogeneous absorption, the hard X-ray energy band is remarkably affected by significant radiation reprocessing, due to scattering into (close to) Compton-thick material surrounding the source, the same material that is causing absorption. The overall X-ray spectrum of V404 Cyg is therefore consistent with the X-ray emission produced by a thick accretion flow, similar to that expected in obscured AGNs accreting at high accretion rates (i.e. close to the Eddington rate), where the emission from the very centre of the system is shielded to the observer.

In figure 5 we show a schematic view of V404 Cyg based on our findings: we argue that the inner part of the accretion flow in V404 Cyg likely takes the form of a slim disk, sustained by strong radiative forces arising from high, possibly (super-)Eddington accretion rates. Such a slim disk is unstable and therefore produces erratically or continuously a cold, clumpy outflow. The material outflowing the source is responsible for both the absorption and reflection features in the spectra, and its variations introduce the remarkable spectral and flux changes observed in the X-ray band. This kind of outflow has never been observed before with such clarity in any accreting BHB.

Interestingly, V404 Cyg is able to produce simultaneously different types of outflows that are normally considered mutually exclusive in other black-hole binaries. Such complex outflows include: (i) a cold, high column-density, non-homogeneous outflow from the inner part of the accretion flow, visible especially below $10 \mathrm{keV}$, in the soft X-rays; (ii) a steady compact jet and fast relativistic ejections, both of which are observed in the radio; (iii) a wind from the outer disk, visible in the optical, where the high opening angle leads to extremely clear P-Cygni profiles, and in the X-rays through narrow line X-ray spectroscopy.

Given the geometric configuration of the system, the intrinsic flux of the source could be at times or even constantly exceeding the Eddington luminosity, while being completely or partially obscured by non-homogeneous material with column densities slightly below or in the Comptonthick regime. This implies that the observed flux might differ even by several orders of magnitude from the source intrinsic flux. Since accretion should work on the same principles in BHBs and AGN, once a suitable scale in mass is applied, detailed studies of V404 Cyg and stellar mass BHs with similar characteristics could help in shading light on some of the inflow/outflow dynamics at play in some, still poorly understood, classes of obscured AGN. 


\section{References}

[1] J. Homan, R. Wijnands, M. van der Klis, T. Belloni, J. van Paradijs, M. Klein-Wolt, R. Fender, and M. Méndez, "Correlated X-Ray Spectral and Timing Behavior of the Black Hole Candidate XTE J1550-564: A New Interpretation of Black Hole States,” ApJ, vol. 132, pp. 377-402, Feb. 2001.

[2] R. P. Fender, J. Homan, and T. M. Belloni, "Jets from black hole X-ray binaries: testing, refining and extending empirical models for the coupling to X-rays," MNRAS, vol. 396, pp. 1370-1382, July 2009.

[3] G. Ponti, R. P. Fender, M. C. Begelman, R. J. H. Dunn, J. Neilsen, and M. Coriat, "Ubiquitous equatorial accretion disc winds in black hole soft states," MNRAS, vol. 422, p. L11, May 2012.

[4] T. M. Belloni and S. E. Motta, "Transient Black Hole Binaries," in Astrophysics of Black Holes: From Fundamental Aspects to Latest Developments (C. Bambi, ed.), vol. 440 of Astrophysics and Space Science Library, p. 61, 2016.

[5] J. Poutanen and A. Veledina, "Modelling Spectral and Timing Properties of Accreting Black Holes: The Hybrid Hot Flow Paradigm,” Space Science Reviews, vol. 183, pp. 61-85, Sept. 2014.

[6] S. Markoff, "From Multiwavelength to Mass Scaling: Accretion and Ejection in Microquasars and AGN," in Lecture Notes in Physics, Berlin Springer Verlag (T. Belloni, ed.), vol. 794 of Lecture Notes in Physics, Berlin Springer Verlag, pp. 143-+, Mar. 2010.

[7] S. Motta, J. Homan, T. Muñoz Darias, P. Casella, T. M. Belloni, B. Hiemstra, and M. Méndez, "Discovery of two simultaneous non-harmonically related quasi-periodic oscillations in the 2005 outburst of the black hole binary GRO J1655-40,” MNRAS, vol. 427, pp. 595-606, Nov. 2012.

[8] M. Revnivtsev, M. Gilfanov, E. Churazov, and R. Sunyaev, "Super-Eddington outburst of V4641 Sgr,” A\&A, vol. 391, pp. 1013-1022, Sept. 2002.

[9] W. R. Morningstar, J. M. Miller, M. T. Reynolds, and D. Maitra, "A Seyfert-2-like Spectrum in the High-mass X-Ray Binary Microquasar V4641 Sgr,” ApJ, vol. 786, p. L20, May 2014.

[10] M. C. Begelman, "Can a spherically accreting black hole radiate very near the Eddington limit," MNRAS, vol. 187, pp. 237-251, Apr. 1979.

[11] M. A. Abramowicz, B. Czerny, J. P. Lasota, and E. Szuszkiewicz, “Slim accretion disks," ApJ, vol. 332, pp. 646-658, Sept. 1988.

[12] S. Fabrika, "The jets and supercritical accretion disk in SS433," Astrophysics and Space Physics Reviews, vol. 12, pp. 1-152, 2004.

[13] K.-y. Watarai, T. Mizuno, and S. Mineshige, "Slim-Disk Model for Ultraluminous X-Ray Sources," ApJ, vol. 549, pp. L77-L80, Mar. 2001.

[14] S. Mineshige, T. Kawaguchi, M. Takeuchi, and K. Hayashida, "Slim-Disk Model for Soft X-Ray Excess and Variability of Narrow-Line Seyfert 1 Galaxies,” PASJ, vol. 52, pp. 499-508, June 2000.

[15] F. Makino, R. M. Wagner, S. Starrfield, M. W. Buie, H. E. Bond, J. Johnson, T. Harrison, and R. D. Gehrz, “V404 Cygni = GS 2023+338,” IAU Circ., vol. 4786, p. 1, May 1989.

[16] J. Casares, P. A. Charles, and T. Naylor, “A 6.5-day periodicity in the recurrent nova V404 Cygni implying the presence of a black hole,” Nature, vol. 355, pp. 614-617, Feb. 1992.

[17] J. C. A. Miller-Jones, P. G. Jonker, G. Nelemans, S. Portegies Zwart, V. Dhawan, W. Brisken, E. Gallo, and M. P. Rupen, "The formation of the black hole in the X-ray binary system V404 Cyg," MNRAS, vol. 394, pp. 1440-1448, Apr. 2009. 
[18] S. D. Barthelmy, A. D’Ai, P. D’Avanzo, H. A. Krimm, A. Y. Lien, F. E. Marshall, A. Maselli, and M. H. Siegel, “Swift trigger 643949 is V404 Cyg.," GRB Coordinates Network, vol. 17929, 2015.

[19] E. Kuulkers, S. Motta, J. Kajava, J. Homan, R. Fender, and P. Jonker, "Renewed activity of V404 Cyg (GS 2023+338)," The Astronomer's Telegram, vol. 7647, p. 1, June 2015.

[20] E. Kuulkers, "Continued public INTEGRAL Target of Opportunity observations of V404 Cyg (GS2023+338),” The Astronomer's Telegram, vol. 7695, June 2015.

[21] E. Kuulkers, "INTEGRAL observations of V404 Cyg (GS 2023+338): public data products," The Astronomer's Telegram, vol. 7758, July 2015.

[22] G. R. Sivakoff, A. Bahramian, D. Altamirano, A. P. Beardmore, E. Kuulkers, and S. Motta, "Swift XRT observations indicates the end of the V404 Cyg 2015 outburst in early August," The Astronomer's Telegram, vol. 7959, p. 1, Aug. 2015.

[23] J. Rodriguez, M. Cadolle Bel, J. Alfonso-Garzón, T. Siegert, X.-L. Zhang, V. Grinberg, V. Savchenko, J. A. Tomsick, J. Chenevez, M. Clavel, S. Corbel, R. Diehl, A. Domingo, C. Gouiffès, J. Greiner, and M. G. H. e. a. Krause, "Correlated optical, X-ray, and $\gamma$-ray flaring activity seen with INTEGRAL during the 2015 outburst of V404 Cygni,” A\&A, vol. 581, p. L9, Sept. 2015.

[24] P. Gandhi, S. P. Littlefair, L. K. Hardy, V. S. Dhillon, T. R. Marsh, A. W. Shaw, D. Altamirano, M. D. Caballero-Garcia, J. Casares, P. Casella, A. J. Castro-Tirado, P. A. Charles, Y. Dallilar, S. Eikenberry, and e. a. Fender, "Furiously fast and red: sub-second optical flaring in V404 Cyg during the 2015 outburst peak,” MNRAS, vol. 459, pp. 554-572, June 2016.

[25] M. Kimura, K. Isogai, T. Kato, Y. Ueda, S. Nakahira, M. Shidatsu, T. Enoto, T. Hori, D. Nogami, C. Littlefield, R. Ishioka, Y.-T. Chen, S.-K. King, C.-Y. Wen, S.-Y. Wang, M. J. Lehner, M. E. Schwamb, and e. a. Wang, J.-H., "Repetitive patterns in rapid optical variations in the nearby black-hole binary V404 Cygni,” Nature, vol. 529, pp. 54-58, Jan. 2016.

[26] F. Bernardini, D. M. Russell, A. W. Shaw, F. Lewis, P. A. Charles, K. I. I. Koljonen, J. P. Lasota, and J. Casares, "Events leading up to the 2015 June Outburst of V404 Cyg," ApJ, vol. 818, p. L5, Feb. 2016.

[27] C. Sanchez-Fernandez, J. J. E. Kajava, S. E. Motta, and E. Kuulkers, "Hard X-ray variability of V404 Cyg during the 2015 outburst," ArXiv e-prints, Aug. 2016.

[28] S. E. Motta, J. J. E. Kajava, C. Sánchez-Fernández, M. Giustini, and E. Kuulkers, “The black hole binary V404 Cygni: a highly accreting obscured AGN analogue,” MNRAS, vol. 468, pp. 981-993, June 2017.

[29] R. Remillard and J. McClintock, "X-Ray Properties of Black-Hole Binaries," Annual Reviews, vol. 44, pp. 49-92, Sept. 2006.

[30] P. T. Życki, C. Done, and D. A. Smith, "The 1989 May outburst of the soft X-ray transient GS 2023+338 (V404 Cyg)," MNRAS, vol. 309, pp. 561-575, Nov. 1999.

[31] T. Oosterbroek, M. van der Klis, B. Vaughan, J. van Paradijs, R. Rutledge, W. H. G. Lewin, Y. Tanaka, F. Nagase, T. Dotani, K. Mitsuda, and K. Yoshida, "On the origin of the iron line in GS 2023+338.," A\&A, vol. 309, pp. 781-786, May 1996.

[32] T. Muñoz-Darias, J. Casares, D. Mata Sánchez, R. P. Fender, M. Armas Padilla, M. Linares, G. Ponti, P. A. Charles, K. P. Mooley, and J. Rodriguez, "Regulation of black-hole accretion by a disk wind during a violent outburst of V404 Cygni,” Nature, vol. 534, pp. 75-78, June 2016. 
[33] N. I. Shakura and R. A. Sunyaev, "Black holes in binary systems. Observational appearance.,” A\&A, vol. 24, pp. 337-355, 1973.

[34] T. Yaqoob, "The nature of the Compton-thick X-ray reprocessor in NGC 4945," MNRAS, vol. 423, pp. 3360-3396, July 2012.

[35] A. P. Beardmore, R. Willingale, E. Kuulkers, D. Altamirano, S. E. Motta, J. P. Osborne, K. L. Page, and G. R. Sivakoff, "Lord of the Rings - Return of the King: Swift-XRT observations of dust scattering rings around V404 Cygni,” MNRAS, vol. 462, pp. 1847-1863, Oct. 2016. 